\title{
Anti-inflammatory effects of 8-hydroxydeoxyguanosine in LPS-induced microglia activation: suppression of STAT3-mediated intercellular adhesion molecule-1 expression
}

\author{
Dong Hyun $\mathrm{Kim}^{1}$, Ik Hyun $\mathrm{Cho}^{3}$, \\ Hong Sook Kim ${ }^{1}$, Joo Eun Jung ${ }^{1}$, \\ Ja Eun Kim ${ }^{1}$, Kwang Ho Lee ${ }^{4}$, \\ Taekyu Park ${ }^{4}$, Young Mok Yang ${ }^{5}$, \\ Seung-Yong Seong ${ }^{2}$, Sang-Kyu $\mathrm{Ye}^{1,6}$ and \\ Myung-Hee Chung ${ }^{1}$
}

\author{
${ }^{1}$ Department of Pharmacology \\ ${ }^{2}$ Department of Microbiology \\ Seoul National University College of Medicine \\ Seoul 110-799, Korea \\ ${ }^{3}$ Department of Physiology \\ College of Dentistry and Dental Research Institute \\ Seoul National University \\ Seoul 110-749, Korea \\ ${ }^{4}$ Department of Biotechnology \\ College of Biomedical and Health Science \\ ${ }^{5}$ Department of Pathology \\ College of Medicine Bio-Food and Drug Research Center \\ Konkuk University \\ Chungju 380-701, Korea \\ ${ }^{6}$ Corresponding author: Tel, 82-2-740-8281; \\ Fax, 82-2-745-7996; E-mail, sangkyu@snu.ac.kr
}

Accepted 22 July 2006

Abbreviations: ChIP, chromatin immunoprecipitation; ICAM-1, intercellular adhesion molecule-1; NAC, N-acetyl-cysteine; oh ${ }^{8} \mathrm{dG}$, 8-hydroxydeoxyguanosine; oh ${ }^{8} \mathrm{Gua}$, 8-hydroxyguanine; ROS, reactive oxygen species; STAT3, signal transducer and activator of transcription 3

\footnotetext{
Abstract

To elucidate the roles of 8-hydroxydeoxyguanosine (oh ${ }^{8} \mathrm{dG}$ ), the nucleoside of 8-hydroxyguanine (oh ${ }^{8} \mathrm{Gua}$ ), we examined the effects of oh $^{8} \mathrm{dG}$ upon LPS-induced intercellular adhesion molecule-1 (ICAM-1) expression and the underlying mechanisms in brain microglial cells. We found that $o^{8} d G$ reduces LPS-induced reactive oxygen species (ROS) production, STAT3 activation, and ICAM-1 expression. ${ }^{\circ}{ }^{8} \mathrm{dG}$ also suppresses pro-inflammatory cytokines, such as TNF- $\alpha$, IL- 6 and IFN- $\gamma$. Overexpression of dominant negative STAT3 completely diminshed STAT3-mediated ICAM-1 transcriptional activity.
}

Chromatin immunoprecipitation studies revealed that ${ }^{8}{ }^{8} \mathrm{dG}$ inhibited recruitment of STAT3 to the ICAM-1 promoter, followed by a decrease in ICAM-1 expression. Using mice lacking a functional Toll-like receptor 4 (TLR4), we demonstrated that, while TLR4+/+ microglia were activated by LPS, TLR4-/microglia exhibited inactivated STAT3 in response to LPS. Evidently, LPS modulates STAT3-dependent ICAM-1 induction through TLR4-mdiated cellular responses. $\mathrm{Oh}^{8} \mathrm{dG}$ apparently plays a role in antiinflammatory actions via suppression of ICAM-1 gene expression by blockade of the TLR4-STAT3 signal cascade in inflammation-enhanced brain microglia. Therefore, $o^{8} \mathrm{dG}$ in the cytosol probably functions as an anti-inflammatory molecule and should be considered as a candidate for development of anti- inflammatory agents.

Keywords: intercellular adhesion molecule-1; microglia; reactive oxygen species; STAT3 transcription factor; Toll-like receptor 4; 8-hydroxydeoxyguanosine

\section{Introduction}

8-hydroxyguanine (oh ${ }^{8} \mathrm{Gua}$ ) is one of the most common DNA lesions generated by reactive oxygen species (ROS) (Kasai et al., 1997). To date, many studies have focused on this oxidized DNA base. However, all of these studies have focused on oh ${ }^{8}$ Gua present in DNA and its mutagenic/carcinogenic action. This action is indicated by induction of $\mathrm{GC} \rightarrow \mathrm{TA}$ transversion due to mismatching with $\mathrm{A}$ (Shibutani et al.,1991), an increase in oh $^{8}$ Gua levels in cellular DNA and urine due to various carcinogens, including (Fraga et al., 1996; Nakae et al., 1997) irradiation (Wilson et al., 1993), pro-carcinogenic inflammatory conditions (Baik et al., 1996), and a reduction in the ${ }^{8}{ }^{8}$ Gua level in cellular DNA and tissue fluids in response to chemopreventive agents (Chung et al., 1992). Therefore, oh $^{8}$ Gua in DNA is not only a mediator of mutagenesis/carcinogenesis, but is also a cause of oxidative stress.

Recently, however, our previous research has indicated that the amount of ${ }^{8}{ }^{8} \mathrm{Gua}$ in cytosol is increased in reaction to strong pathogenic infections and that oh $^{8}$ Gua can not be incorporated into DNA. 
When present within DNA, oh ${ }^{8} \mathrm{Gua}$ induces other base damage, whereas when ${ }^{8}{ }^{8}$ Gua exists as a separate molecule outside of DNA, it protects normal bases and DNA under oxidation conditions (Kim et al., 2006). The protective action of $\mathrm{oh}^{8} \mathrm{Gua}$ was much stronger than the actions of well known ROS scavengers, such as manitol, DMSO, t-butyl alcohol, azide, and formate, indicating that oh $^{8} \mathrm{Gua}$ is a strong antioxidant. The strong antioxidant action of $\mathrm{oh}^{8} \mathrm{dG}$ was also confirmed by the observation that oxidation of dichlorodihydrofluorecein by peroxynitirite is most severely inhibited by $0 h^{8} \mathrm{dG}$ among antioxidants tested (Kim et al., 2004). Many naturally occurring and synthetic antioxidants are known to have an anti-inflammatory effect and many of them are used for treatment or prevention of various inflammatory disorders, such as rheumatoid arthritis (Choi et al., 2003), atherosclerosis (Shimosawa et al., 2005), diabetic complications (Kocak et al., 2000), and inflammatory bowel diseases (Dryden et al., 2005). Therefore, the antioxidant property of $\mathrm{oh}^{8} \mathrm{dG}$ should contribute to the anti-inflammatory effect for treatment of neuroinflammatory-related diseases. However, the molecular mechanisms by which $\mathrm{oh}^{8} \mathrm{dG}$ exerts its anti-inflammation effects in brain microglial cells are largely unknown.

ICAM-1 is an inducible surface glycoprotein belonging to the immunoglobulin superfamily that is involved in a wide range of inflammatory and immune responses (Butcher et al., 1996). During inflammation, ICAM-1 can be dramatically upregulated by bacterial lipopolysaccharid (LPS), phorbol esters, and inflammatory cytokines, such as TNF- $\alpha, I L-1 \beta$, and IFN- $\gamma$. (Wahl et al., 1996). The ICAM-1 promoter contains the STAT1/3 binding motif (GAS) sequence or the interferon response element (IRE). ROS, including hydrogen peroxide $\left(\mathrm{H}_{2} \mathrm{O}_{2}\right)$ and superoxide, and pro-inflammatory cytokine, such as interleukin-6 (IL-6) and IFN- $\gamma$, are known to induce ICAM-1 transcription and inflammatory responses (Caldenhoven et al., 1994; Kim et al., 2002). The JAK/STAT signaling pathway has been reported to mediate growth factor actions, hormones, and cytokines (Ihle et al., 2001). Recently, several studies have reported that JAK/STAT signaling mediates inflammatory responses in brain microglial cells (Kim et al., 2003; Kim et al., 2005). However, the precise roles of $\mathrm{oh}^{8} \mathrm{dG}$ in the JAK/STAT signaling pathway and ICAM-1 expression remain to be clarified.

Microglial cells are the resident immune cells of the brain that secrete proinflammatory factors and ROS in response to immunological stimuli. Overresponsiveness or lack of regulation of microglia activation results in neurotoxic consequences (Liu et al., 2003). Activated microglia are observed in various neurological diseases, multiple sclerosis, and Alzheimer's disease (Kreutzberg et al., 1996). The Toll-like receptor 4 (TLR4) is traditionally accepted as the primary (LPS) receptor (Lien et al., 2000) and has been reported as critical for the microglial response to LPS (Lehnardt et al., 2002). Thus, it may be functionally important to tightly regulate the degree of microglial activation after injury and during inflammatory disease.

In this study, we provide novel evidence that the anti-inflammatory actions of $\mathrm{oh}^{8} \mathrm{dG}$ in the cytosol result from inhibition of ICAM-1expression via the TLR4-STAT3 signal cascade and a ROS dependent mechanism in brain microglia. These findings indicate that ${ }^{8}{ }^{8} \mathrm{dG}$ in the cytosol functions as an anti-inflammatory molecule and that STAT3-dependent repression of inflammatory gene expression may be used to control an excess immune response in various inflammatory diseases. Therefore, the observed anti-inflammatory functions of oh ${ }^{8} \mathrm{dG}$ indicate that it is a candidate for use in treatment of neuroinflammatory diseases.

\section{Materials and Methods}

\section{Reagents}

$\mathrm{Oh}^{8} \mathrm{dG}, \quad 3-[4,5$-dimethylthiazol-2-yl]-2,5-diphenyltetrazolium bromide (MTT), and LPS were purchased from Sigma (St. Louis, MO). Antibodies to phosphorylated STAT3, total STAT3, $\beta$-actin, and ICAM-1 were obtained from Santa Cruz Biotechnology (Santa Cruz, CA) and rat anti-OX-42 (CD11b) was purchased from Serotec. Transfection reagents Lipofectamine were products of Invitrogen Life Technologies (Carlsbad, CA). Fc Oxyburst green assay reagent was purchased from Molecular Probe, Inc.

\section{Cell culture and MTT assay}

BV2 immortalized murine microglial cells were obtained from Dr. Suck K.H. (Kyungpook National University). The BV2 cell line was grown in DMEM and supplemented with $5 \%$ fetal bovine serum. Cells were serum-starved overnight before treatment with LPS $(100 \mathrm{ng} / \mathrm{ml})$. The effect of $\mathrm{oh}^{8} \mathrm{dG}$ on the viability of microglial BV2 cells was determined using an MTT assay.

\section{Cytokine ELISA}

The production of cytokines was determined by a cytokine-specific ELISA analysis of culture supernatants. A matched antibody pair from PharMingen (Hamburg, Germany) was used according to the manufacturer's instructions for TNF- $\alpha$, IL- 6 and IFN- $\gamma$. 


\section{Animals and stereotaxic injection}

The intrastriatal administration of drugs was modified from a previously described method (Heneka et al., 2000; Park et al., 2006). Briefly, male C57BL/6 mice (approx. weighing 22-25 g) were anesthetized by intraperitoneal (i.p.) injection with sodium pentobarbital $(30 \mathrm{mg} / \mathrm{kg})$, and placed on a stereotaxic apparatus (Myneurolab, St. Louis, MO). Mice were injected with PBS or oh ${ }^{8} \mathrm{dG}(8.0 \mu \mathrm{g}$ in $2.0 \mu \mathrm{l}$ of PBS) or LPS $(0.5 \mu \mathrm{g}$ in $1.0 \mu \mathrm{l}$ of PBS) into the right striatum (stereotaxic coordinates in $\mathrm{mm}$ with reference to bregma: $\mathrm{AP},+0.7 ; \mathrm{ML},+2.0 ; \mathrm{DV},-3.5)$ or the right hippocampus (AP, -2.7 ; $\mathrm{ML},+2.5 ; \mathrm{DV},-3.0$ ) using a $10 \mu \mathrm{l}$ Hamilton microsyringe with a 26-gauge needle. $\mathrm{Oh}^{8} \mathrm{dG}$ was administrated by intrastriatal or hippocampal injection $30 \mathrm{~min}$ before LPS injection. Control mice received an equal volume of PBS vehicle. The incision was cleaned with saline and sutured. All surgical and experimental procedures were reviewed and approved by the Institutional Animal Care and Use Committee (IACUC) in the College of Medicine, Seoul National University.

\section{Immunohistochemical analysis}

After deep anesthesia, the mice were perfused with fresh $4 \%$ paraformaldehyde in $0.1 \mathrm{M}$ phosphate buffer ( $\mathrm{pH}$ 7.4). Brains were removed and postfixed at $4^{\circ} \mathrm{C}$ overnight, transferred to $30 \%$ sucrose in PBS for $48 \mathrm{~h}$, and then coronal sections ( $30 \mu \mathrm{m}$ thickness) were prepared on a cryostat. The immunohistochemical analysis of floating sections was performed as previously described (Park et al., 2006). Briefly, sections were incubated for 30 min with $3 \% \mathrm{H}_{2} \mathrm{O}_{2}$ in $0.1 \mathrm{M}$ PBS $(\mathrm{pH} 7.4)$, then blocked with a solution containing 5\% normal goat/or horse serum, $2 \%$ BSA, $2 \%$ FBS, and $0.1 \%$ triton $\mathrm{X}-100$ for $2 \mathrm{~h}$ at room temperature (RT). The sections were incubated overnight at $4^{\circ} \mathrm{C}$ with mouse anti-phospho STAT3 (1:500), mouse anti-ICAM-1 (1:500; Santa Cruz biotechnology, Santa Cruz, CA), or rat anti-OX-42 (CD11b) (1:50; Serotec). The sections were then incubated with biotinylated secondary antibodies (1:200; Vector Labs., Burlingame, CA) for $1 \mathrm{~h}$ at RT, followed by incubation with an avidin and biotinylated HRP complex (1:200; Vector Labs., Burlingame, CA) for $1 \mathrm{~h}$ at RT. All sections were visualized with 3, 3'-diaminobenzidine. The sections were mounted onto gelatin-coated slide glass, and coverslips were applied with permount. Images were captured using an image system (KAPPA ImageBase DX 30, KAPPA Opto-electronic Inc). For immunofluorescence staining, floating sections were incubated overnight at $4{ }^{\circ} \mathrm{C}$ with a mixture of mouse anti-phospho STAT3 antibody (1:500) and rat anti-OX-42 antibody (1:50). The sections were then incubated for $1 \mathrm{~h}$ at RT with a mixture of TRITC- and FITC-conjugated mouse/rabbit IgG antibody (1:200; Jackson ImmunoResearch). The sections were mounted with VectaShield (Vector Labs., Burlingame, CA). The double-stained images were analyzed using confocal laser scanning microscopy (LSM 5 PASCAL; Carl Zeiss, Germany).

\section{ROS production assay}

BV2 cells were plated at $2 \times 10^{6}$ cells $/ \mathrm{ml}$ and serumstarved overnight. The Green assay reagent (Molecular Probes) consisted of bovine serum albumin (BSA) that was covalently linked to dichlorodihydroflurescein $\left(\mathrm{H}_{2} \mathrm{DCF}\right)$ and complexed with purified rabbit polyclonal anti-BSA IgG antibodies. Before initiating the reaction by adding Fc-Oxyburst, BV2 cells were preincubated with each reagent, a potent antioxidant compound, $\mathrm{N}$-acetyl-cysteine (NAC), and oh $^{8} \mathrm{dG}$ for $1 \mathrm{~h}$. Fc-Oxyburst immune complexes $(120 \mu \mathrm{g} / \mathrm{ml})$ were then added followed by incubation for $30 \mathrm{~min}$ at $37^{\circ} \mathrm{C}$ with LPS. Fluorescence was measured using FACSCaliber flow cytometry (Becton Dickinson, Mountain View) at $530 \mathrm{~nm}$.

\section{Western blot analysis}

Cell lysates were separated by SDS-PAGE and transferred to nitrocellulose membranes. Membranes were incubated with primary antibodies and peroxidase-conjugated secondary antibodies (Zymed, San Francisco, CA) and then visualized using an ECL system.

\section{RT-PCR}

Cellular RNAs were extracted using TRIZOL reagents (Invitrogen, Carlsbad, CA). RT-PCR was performed in a final volume of $25 \mu$ using a Super Script One-step RT-PCR kit (Invitrogen, Carlsbad, $\mathrm{CA}$ ) in an automated thermal cycler (GeneAmp PCR system 9700, Perkin Elmer, Norwalk Ct). PCR assays were performed over 32 cycles consisting of denaturation at $94^{\circ} \mathrm{C}$ for $30 \mathrm{~s}$, annealing at $56^{\circ} \mathrm{C}$ for $40 \mathrm{~s}$, and extension at $72^{\circ} \mathrm{C}$ for $1 \mathrm{~min}$. PCR products were analyzed in $1.7 \%$ agarose gel containing ethidium bromide $(\mathrm{EtBr})$. The sequences of PCR primers were reverse, 5'-CAGGGAAGAATCTGGAAAGGT--3', and forward, 5'-AAATGGGCTTTCCGAATTCA--3', for TNF- $\alpha$; reverse, 5'-AAGTGCATCATCGTTGTTCATACA--3', and forward, 5'-GAGGATACCACTCCCAACAGACC--3', for IL-6; reverse, 5'AAATTAGTTATGAATCCTTTCTGTCATC-3', and forward, 5'-ACAACACCATGGGCCCGCAGGCGGC-3', for IFN- $\gamma$. 


\section{Luciferase reporter assay}

Dominant-negative mutants of the STAT3 and ICAM-1 luciferase reporter constructs were obtained from Dr. M Shong (Chungnam National University). Transient transfections were performed in duplicate in $35 \mathrm{~mm}$ dishes using Lipofectamine reagents as instructed by the manufacturer (Invitrogen Life Technologies). To normalize for variations in cell number and transcription efficiency, all clones were cotransfected with pCMV- $\beta$-galactosidase for $24 \mathrm{~h}$. A luciferase assay was performed according to the manufacturer's instructions (Promega, Madison, WI) using $20 \mu \mathrm{l}$ of cell extract in $100 \mu \mathrm{l}$ of assay buffer. The light intensity was measured for $30 \mathrm{~s}$ on a Luminometer (Berthold Lumat LB9501; Berthold Tedhnologies, Oak Ridge, TN). The luciferase activity was normalized by measurement of the $\beta$-galactosidase activity (in $\mathrm{OD}^{420}$ ).

\section{Chromatin immunoprecipitation assay}

A chromatin immunoprecipitation (ChIP) assay was performed as described previously (Ye et al., 2001). $\mathrm{oh}^{8} \mathrm{dG}$ treated BV2 cells were fixed in formaldehyde. Soluble chromatin samples were immunoprecipitated with anti-STAT3 antibody at $4^{\circ} \mathrm{C}$ for $3 \mathrm{~h}$. DNA isolated from immunoprecipitated material following reversal of formaldehyde crosslinking was amplified by PCR. Promoter-specific primers included mouse ICAM-1, 5'-ACTCACCTGCTGGTCTCTGA-3' for sense and 5'-CGCTTTTATAGTCTCTGGCT-3' for antisense, which amplified 230 bp fragments flanking the STAT3 binding element.

\section{Statistical analyses}

Statistical analyses were performed using Student's t-test. Data were considered statistically significant when $P$ was less than 0.05 .

\section{Results}

\section{Brain BV2 microglial cells with ${ }^{8}{ }^{8} \mathrm{dG}$ show almost} no change in viability

To determine the toxicity of $o h^{8} d G$, we first examined cell viability using the MTT test in BV2 cells. After $72 \mathrm{~h}$ of incubation, cell viability was determined. As shown in Figure 1A, the viability of BV2 cells was not affected by oh ${ }^{8} \mathrm{dG}(0-100 \mu \mathrm{g} / \mathrm{ml})$. After confirmation of the pharmacologic effects, a toxicological study of ${ }^{8}{ }^{8} \mathrm{dG}$ was performed to examine possible side effects.

\section{$\mathrm{Oh}^{8} \mathrm{dG}$ inhibits LPS-elicited production of proin- flammtory cytokines in microglial BV2 cells}

In order to determine the functional significance of the $\mathrm{oh}^{8} \mathrm{dG}$-mediated decrease in proinflammtory cytokines, oh ${ }^{8} \mathrm{dG}$-treated BV2 cells were prepared
A

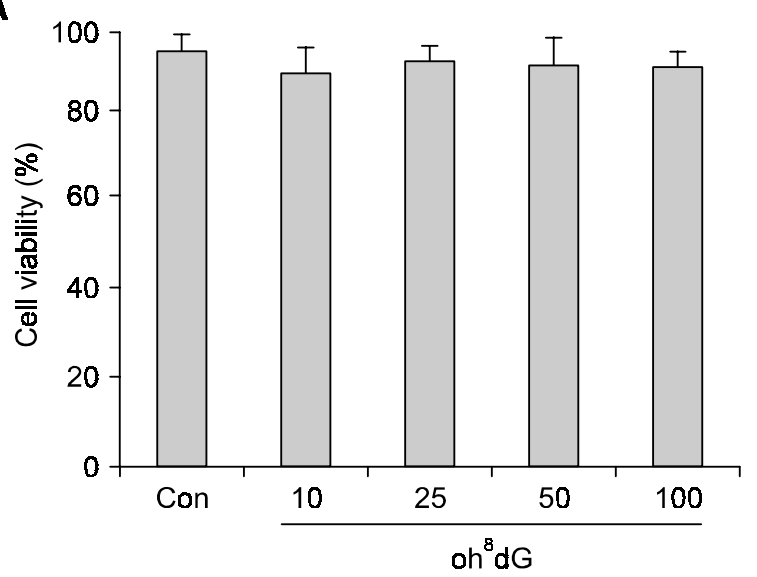

B

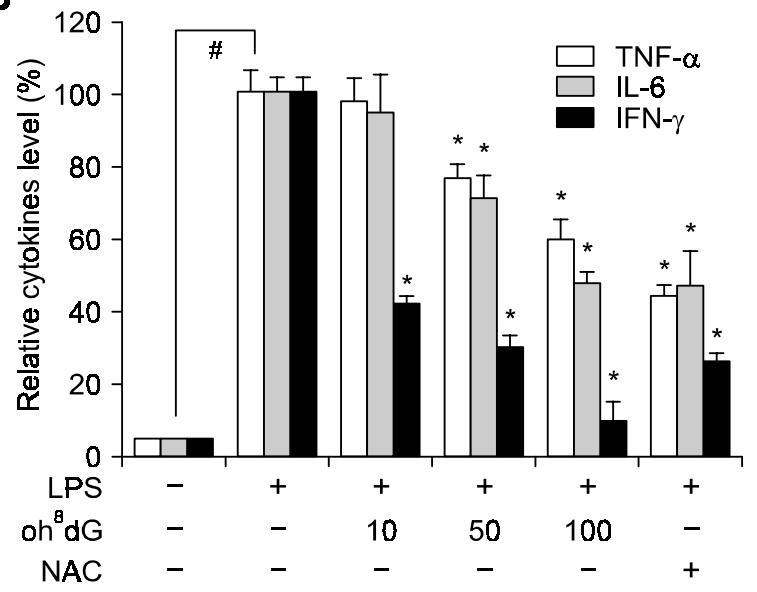

C

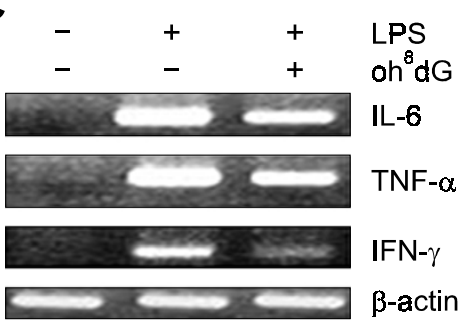

Figure 1. Intrastriatal $\mathrm{h}^{8} \mathrm{dG}$ administration attenuates LPS-induced inflammatory responses in the brain. (A) Examination of oh ${ }^{8} \mathrm{dG}$ toxicity in BV2 brain microglial cells by MTT assay. BV2 microglial cells were treated with various concentrations of oh ${ }^{8} \mathrm{dG}(0-100 \mu \mathrm{g} / \mathrm{ml})$ for 3 days and cell viability was examined by MTT assay. (B) Effect of oh ${ }^{8} \mathrm{dG}$ on the expression of pro-inflammatory cytokines in BV2 cells. Cytokines in the culture supernatants were analyzed by ELISA. Values represent mean \pm S.E.M. $(n=5) .{ }^{\#} P<0.01$ vs. medium alone-treated group. ${ }^{\sharp} P<0.01$ vs. medium alone-treated group. ${ }^{*} P<0.01$ vs. LPS alone-treated group. (C) IL-6, TNF- $\alpha$, and IFN- $\gamma$ mRNA levels were also analyzed to determine the effects of oh ${ }^{8} \mathrm{dG}$ in cytokine production. The transcription of $\beta$-actin was measured for normalization. 
and examined. The cells were treated with LPS alone or with various concentrations of $\mathrm{oh}^{8} \mathrm{dG}$ for 24 h. The concentrations of cytokines in the culture supernatant of BV2 cells were measured by ELISA. As shown in Figure 1B, the amounts of pro-inflammtory cytokines, such as TNF- $\alpha$, IL- 6 , and IFN- $\gamma$ were increased in the culture medium after LPS stimulation but these increases were suppressed by $\mathrm{oh}^{8} \mathrm{dG}$ in a dose-dependent manner. RT-PCR analysis showed that $\mathrm{oh}^{8} \mathrm{dG}$ suppressed LPS-in- duced IL-6, TNF- $\alpha$ and IFN- $\gamma$ mRNA expression (Figure $1 \mathrm{C}$ ). Thus, $\mathrm{oh}^{8} \mathrm{dG}$ also inhibits the release of pro-inflammatory cytokines, thus contributing to efficient prevention of inflammation.

\section{$\mathrm{Oh}^{8} \mathrm{dG}$ attenuates LPS-induced brain inflammation} A mouse model of brain inflammation due to intrastriatal (i.s.) injection of LPS $(0.5 \mu \mathrm{g} / 1.0 \mu \mathrm{l}$ in PBS) was established to evaluate the anti-inflam-

A

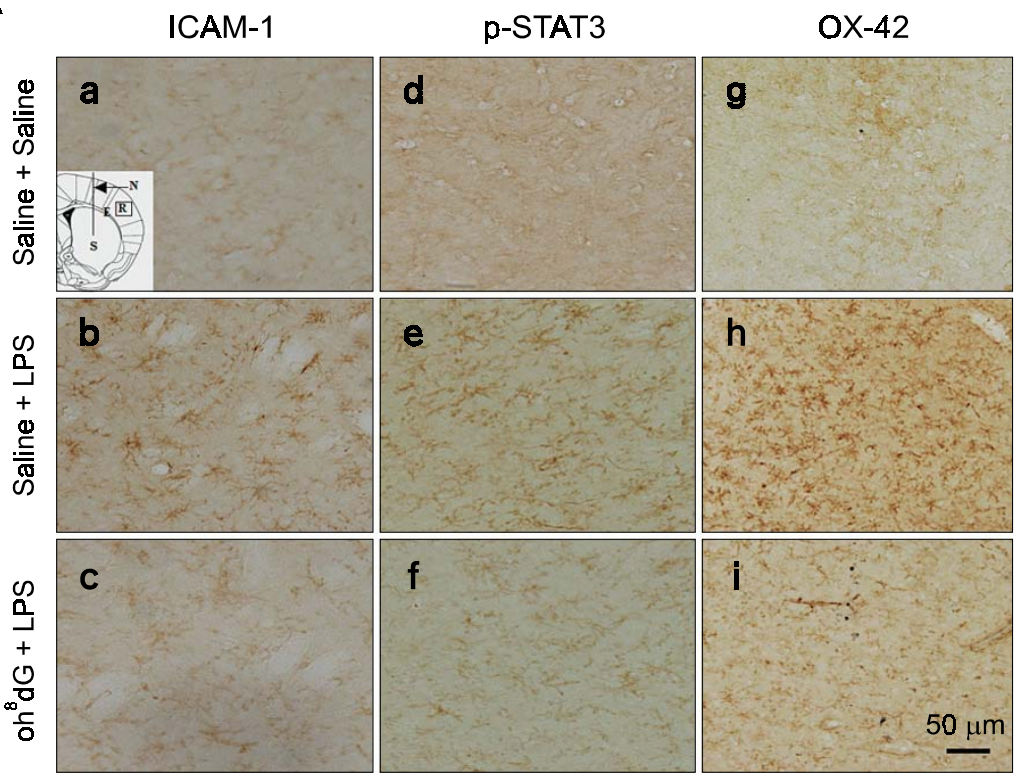

B

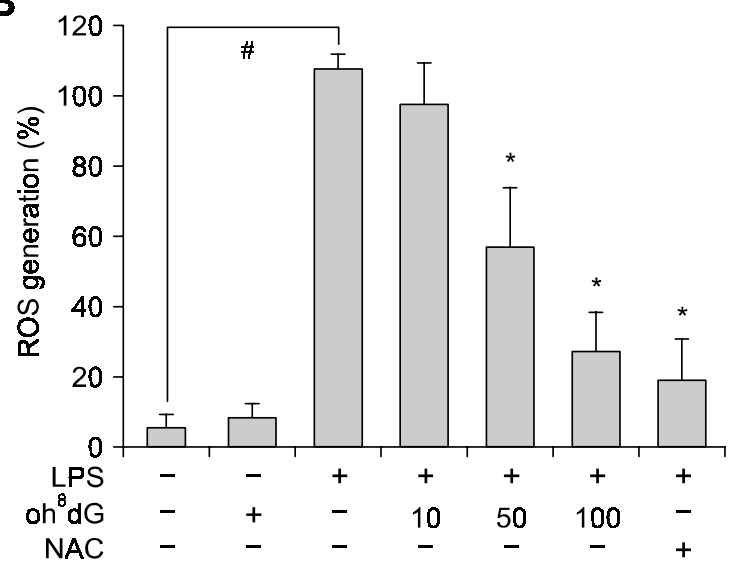

C

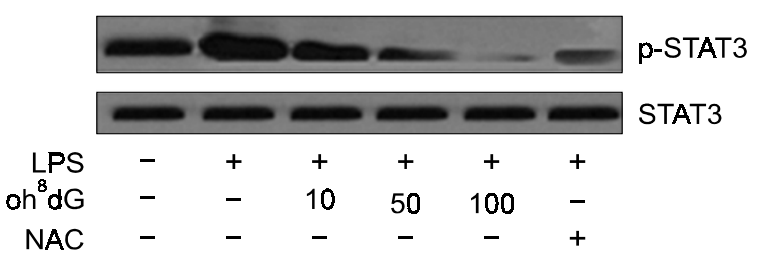

Figure 2. Intrastriatal oh $^{8} \mathrm{dG}$ administration suppresses LPS-induced microglia activation. (A) Immunostained cortical sections 1 day after intrastriatal injections of saline + saline $(a, d, g)$, saline $+1.0 \mu \mathrm{g}$ of LPS $(b, e, h)$, or $8.0 \mu g$ of oh ${ }^{8} d G+1.0 \mu g$ of LPS (c, f, i). LPS-induced ICAM1 $(a-c), p-S T A T 3(d-f)$ activation and OX-42 (g-i) were markedly suppressed by intrastriatal oh ${ }^{8} \mathrm{dG}$ administration. Photomicrographs show the marked regions (rectangles) of the insets at the right, bottom. Bar $=50 \mu \mathrm{m}$. (B) BV2 cells grown in 96 well plates were treated with LPS and the Fc-oxyburst immune complex in the absence and presence of $1 \mathrm{~h}$ pre-added oh ${ }^{8} \mathrm{dG}$, NAC. The fluorescence values were measured using FACS Calibur flow cytometry. Values are mean \pm S.E.M. $(n=4) .{ }^{\#} P<0.01$ vs. medium alone-treated group. ${ }^{*} P<0.01$ vs. LPS alone-treated group. (C) BV2 cells were pretreated with oh ${ }^{8} \mathrm{dG}$, NAC, for $1 \mathrm{~h}$, and then stimulated with LPS for $1 \mathrm{~h}$. Cell lysates were separated by $8-10 \%$ SDS-PAGE, and western blots were probed with anti-phospho-STAT3 and STAT3. 
matory effects of $\mathrm{oh}^{8} \mathrm{dG}$, which was administered at a dose of $8.0 \mu \mathrm{g} / 2 \mu \mathrm{l}$ in PBS $1 \mathrm{~h}$ prior LPS-i.s. injection. The i.s. injection of LPS resulted in inflammation in the forebrain cortex and striatum, which was comparable to the cortex and striatum inflammation induced by i.s. injection of LPS in mice as described by Heneka et al. (2000). The level of brain inflammation was assessed after 1 day of
LPS-i.s. injection (Figure 2A). Figure 2A shows changes in expressions of inflammatory markers (ICAM-1). PBS- or oh ${ }^{8}$ dG-treated mice showed resting, ramified microglia-like cells with fine delicate processes that were marked by ICAM-1, p-STAT3, and OX-42 antibodies (Figure 2A-a, d, g). In LPS-treated mice, ICAM-1, p-STAT3, and OX-42-immunoreactive (IR) cells were detected in the cortex (Figure 2A-b,

A

pCAM-175
GAS $(\mathrm{m})$

B

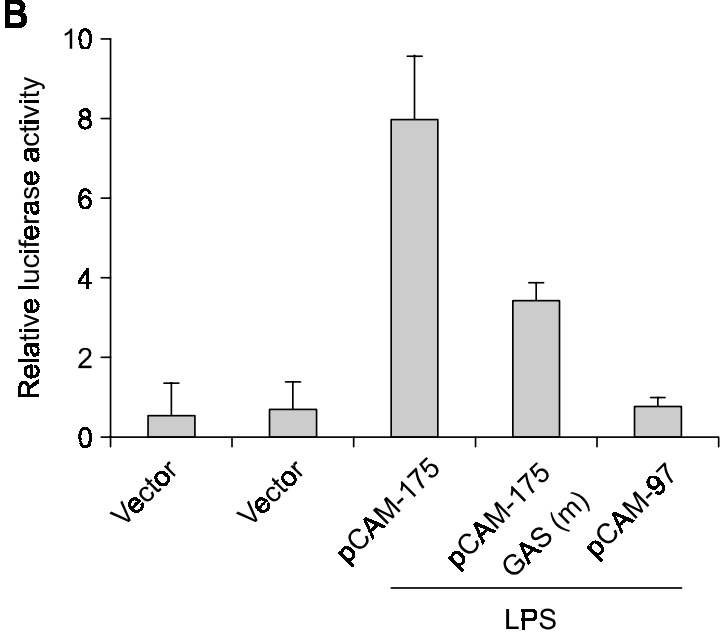

D

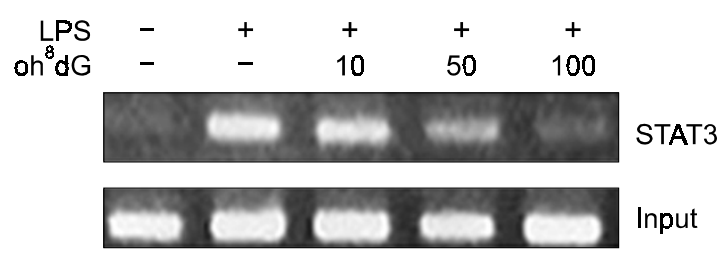

C

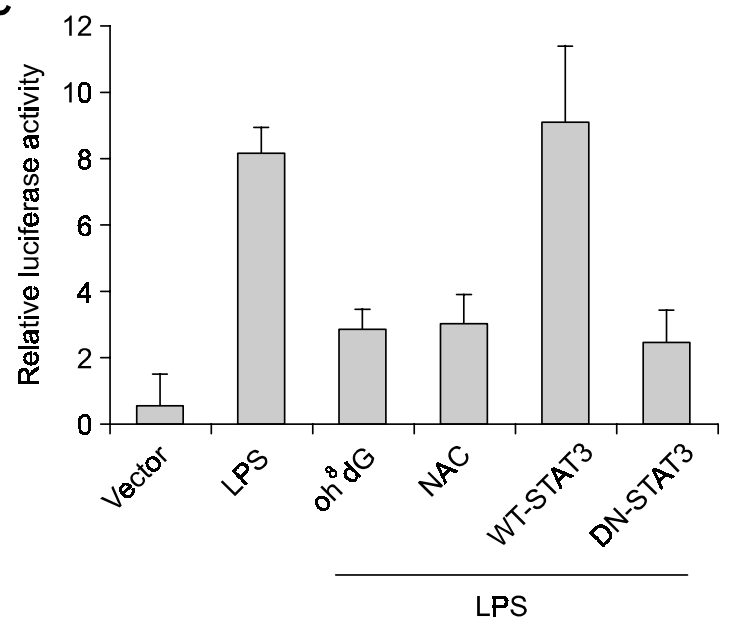

E

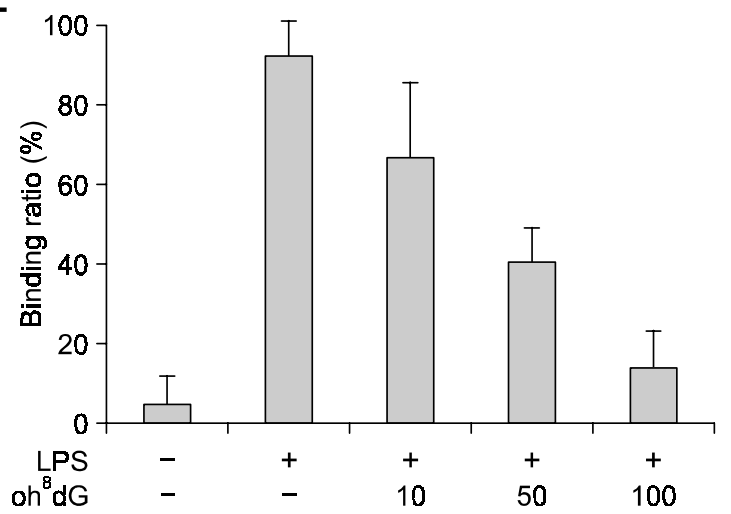

Figure 3. Effects of oh ${ }^{8} \mathrm{dG}$ on the promoter activity of ICAM-1 in BV2 cells. (A) Schematic representation of the ICAM-1 reporter genes used. (B, C) The ICAM-1 luciferase construct was cotransfected with wild type STAT3 (WT-STAT3), dominant negative STAT3 (DN-STAT3), or vector alone into BV2 cells for $24 \mathrm{~h}$. The cells were pretreated with oh ${ }^{8} \mathrm{dG}$, NAC for $1 \mathrm{~h}$, and then stimulated with LPS for $24 \mathrm{~h}$. The luciferase activities shown in this study were calculated following measurement of the $\beta$-galactosidase activity to normalize for transfection efficiency. Bars represent the average luciferase activity calculated from four independent experiments. (D, E) ChIP assay. Soluble chromatin preparation from BV2 cells after oh ${ }^{8} \mathrm{dG}$ treatment caused immunoprecipitation with the STAT3 antibody. Purified DNA was amplified by semiquantitative PCR using primers spanning the ICAM-1 promoter sequences. Five-fold dilutions of total BV2 DNA (Input) were amplified as an internal control for PCR. 
e, h) and the striatum (data not shown) in the ipsilateral hemisphere. $\mathrm{oh}^{8} \mathrm{dG}$ clearly reduced the number of ICAM-1, p-STAT3, and OX-42-IR cells (Figure 2A-c, f, i). These results indicate that $o^{8} d G$ has an anti- inflammatory effect in brain microglia.

\section{$\mathrm{Oh}^{8} \mathrm{dG}$ reduces LPS-induced ROS production}

To study the effect of $\mathrm{oh}^{8} \mathrm{dG}$ on ROS production, we measured the release of ROS using Fc-oxyburst. Release of ROS can be detected approximately 30 min after LPS treatment in BV2 microglia, and cells were treated with $\mathrm{oh}^{8} \mathrm{dG}$ and NAC $1 \mathrm{~h}$ before LPS exposure. As shown in Figure $2 \mathrm{~B}$, $\mathrm{oh}^{8} \mathrm{dG}$ reduced LPS-induced ROS production, similar to results with NAC. Therefore, there exists a possibility that the inhibition of ROS production by $\mathrm{oh}^{8} \mathrm{dG}$ may be due to the ROS scavenging action of $o^{8} \mathrm{dG}$. To further clarify that these inhibitory effects occur via STAT3 phosphorylation, we also examined the activation of STAT3. Effects similar to oh $^{8} \mathrm{dG}$ for STAT3 were observed in BV2 microglial cells (Figure 2C). This indicates that $\mathrm{oh}^{8} \mathrm{dG}$ reduced the activation of STAT3, suggesting that STAT3-dependent inflammatory signaling is related to the anti-inflammatory activity of $\mathrm{oh}^{8} \mathrm{dG}$.
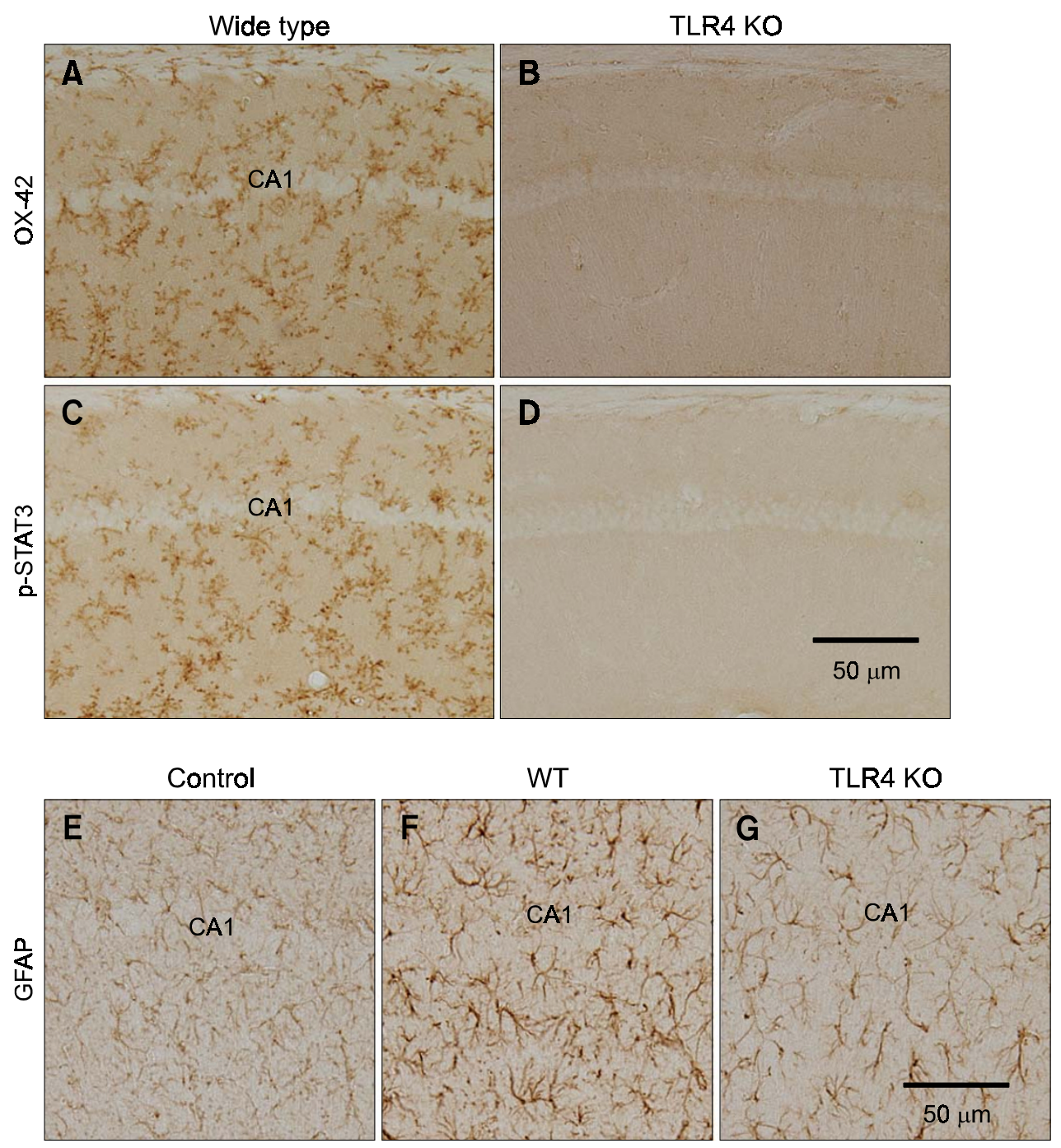

Figure 4. LPS-induced inflammation was mediated by TLR4. (A-D) Immunostained sections 1 day after intrahippocampal injections of $0.5 \mu \mathrm{g}$ of LPS in the wild type (A, C) and TLR 4 knock-out mice (B, D). Numbers of OX-42 and p-STAT3 cells were significantly increased in the hippocampus of wild type mice, but not TLR4 knock-out mice. Astrocytes and GFAP IR cells were also significantly activated in the hippocampus of LPS-injected wild type mice $(F)$ compared with $E$, however astroglial activation was notably attenuated by TLR4 deletion $(G)$. Scale bar $=50 \mu \mathrm{m}$. 


\section{$\mathrm{Oh}^{8} \mathrm{dG}$ suppresses LPS-induced ICAM-1 promoter activity via STAT3}

We examined the possibility that ICAM-1 expression is controlled by STAT3-dependant transcriptional regulation. Figure $3 \mathrm{~A}$ shows a schematic diagram of the deletion mutant constructs indicating the location of GAS, Sp-1, and TATA box sites. Mutation of the GAS sequence, pCAM-175 GAS $(\mathrm{m})$ (see Figure $3 A$ ), eliminated LPS-dependent promoter activity (Figure 3B). To evaluate the contribution of STAT3 mediation of cellular signaling in activated microglia, we established dominant-negative mutant cells transiently expressing dominant negative (DN) STAT3. $\mathrm{Oh}^{8} \mathrm{dG}$ inhibited LPS-enhanced ICAM-1 promoter activity, and NAC also reduced promoter activity, indicating that ICAM-1 gene expression is STAT3 and ROS dependent (Figure 3C). We also evaluated the in vivo effects of $\mathrm{oh}^{8} \mathrm{dG}$ on the interaction of STAT3 and the ICAM-1 promoter region. A ChIP assay was performed using cells that were treated for $12 \mathrm{~h}$ with either LPS or $\mathrm{oh}^{8} \mathrm{dG}$. Results shown in Figure 3D and $\mathrm{E}$ demonstrate that the amount of the ChIP complex was dramatically reduced by $\mathrm{oh}^{8} \mathrm{dG}$. Thus, $\mathrm{oh}^{8} \mathrm{dG}$ inhibits LPSinduced ICAM-1 expression and STAT3 binding activity in BV2 cells.

\section{LPS-induced microglia activation in the absence of TLR4}

To investigate the inflammatory process in the LPS-injected brain, the activations of microglia, STAT 3 , and astrocytes in the hippocampus were analyzed using immunostaining. Anti-OX-42, a marker for microglia/macrophage lineage cells, was used for microglia, anti-phosho-STAT 3 (p-STAT 3) was used for STAT 3, and anti-GFAP, a marker for astrocytes, was used for astrocytes. In LPS-injected wild type mice, the number of OX-42 and p-STAT 3 IR cells (the intensely activated form with an enlarged cell body and much shorter and thicker) was remarkably increased in both the CA1 and CA3 regions of the ipsilateral hippocampus (Figure 4A, C). OX-42 and p-STAT 3 IR cells were hardly detected in the hippocampus of LPS-injected TLR4 knock out mice (Figure 4B, D). Immunostaining for GFAP was used as a positive control for astrocytes. GFAP IR cells were also significantly activated in the hippocampus of LPS-injected wild type mice (Figure 4F, compared with Figure 4E), however astroglial activation was notably attenuated by TLR4 deletion (Figure 4G). Therefore, LPS-induced microglial activation is probably mediated, at least in part, by TLR4-STAT3 dependent responses. While several other potential LPS receptors and pathways exist (Perera et al., 2001), future research will need to focus on the effects of oh $^{8} \mathrm{dG}$ in TLR4-dependent pathways.

\section{Discussion}

While the harmful effects of oh ${ }^{8}$ Gua present in DNA have been studied extensively, there have been few reports about the effects of oh $^{8} \mathrm{Gua}$ on cellular functions in the cytosol. Therefore, we were interested in the effects of oh $^{8} \mathrm{dG}$ because cell membranes are permeable to this nucleoside and the effects of oh ${ }^{8} \mathrm{dG}$ can be evaluated both in vitro and in vivo. This study, for the first time, reveals that the anti-inflammatory actions of $\mathrm{oh}^{8} \mathrm{dG}$ are related to inhibition of STAT3 activation, a mediator involved in LPS signaling and inflammation. We have demonstrated that $\mathrm{oh}^{8} \mathrm{dG}$, as an anti-oxidant, inhibits ROS production and secretion of inflammatory cytokines in LPS-stimulated microglial cells. oh $^{8} \mathrm{dG}$ can be developed as a potent anti-inflammatory agent that can inhibit ICAM-1 expression in inflammatory diseases.

A number of studies have reported that 8hydroxyguanine (oh ${ }^{8} \mathrm{Gua}$ ), one of the most common DNA lesions generated by ROS, causes mutagenic/ carcinogenic effects. However, our previous observations indicate that ${ }^{8}{ }^{8} \mathrm{dG}$ is an antioxidant, based on easy degradation of $\mathrm{oh}^{8} \mathrm{dG}$ compared to normal nucleosides ( $d A, d G, d C, d T$ ). oh ${ }^{8} d G$ was rapidly degraded when exposed to various ROS, indicating that oh $^{8} \mathrm{dG}$ easily interacts with ROS and is an efficient ROS scavenger. oh ${ }^{8} \mathrm{Gua}$ also has different functions when present in DNA and when not contained within DNA. Although oh ${ }^{8} \mathrm{Gua}$ is harmful when present in DNA, it can be beneficial when it exists as a separate molecule. $\mathrm{Oh}^{8} \mathrm{Gua}$ within DNA is rapidly released by 8-oxoguanine glycosylase 1 (OGG1). This released oh $^{8}$ Gua protects intermolecular DNA from oxidative stress, acting as antioxidant when it exists as a separate molecule (Kim et al., 2004). Antioxidant agents have antiinflammatory effects because ROS, produced during inflammatory action by phagocytic immune cells, cause the death of pathogens (Ahluwalia et al., 2004). In agreement with studies of ROS production, oh $^{8} \mathrm{dG}$ abolishes LPS-elicited ROS production in a dose-dependent manner (Figure 2B). These inhibitory effects were also observed following addition of NAC, which markedly suppressed LPS- induced pro-inflammatory cytokine release (Figure 1B, C), STAT3 activity, and expression of ICAM-1 (Figure 2A). ICAM-1 with its counter receptor, the lymphocyte function-associated antigen 1 (LFA-1), are known to play key roles in inflammatory processes and in T-cell mediated host defense mechanisms.

The ICAM-1 promoter contains a signal transducer and activator of transcription [1(STAT1)/STAT3 binding motif (GAS sequence)] that flanks the specificity 
protein 1 (Sp1) binding site, and a promoter cisacting sequence (TATA) box. The JAK-STAT cascade is a regulator of various genes involved in immune and inflammatory responses (Stratowa et al., 1995). Although STAT proteins were discovered during the analysis of interferon signaling, recent studies have revealed that STAT signaling can account for various cellular responses to a number of cytokines, growth factors, and hormones $(\mathrm{Wu}$ et al., 1997). ROS, $\mathrm{H}_{2} \mathrm{O}_{2}$ and superoxide and proinflammatory cytokines, such as IL-6 and IFN- $\gamma$, are known to induce transcriptional complexs that bind to the ICAM-1 GAS sequence (Simon et al., 1998). Mutation or deletion of this element decreases ICAM-1 promoter activity (Caldenhoven et al., 1994). The Sp1-STAT3 complex appears to play an important role in upregulation of ICAM-1 transcription after reoxygenation or reperfusion (Yang et al., 2005).

STAT3 was first identified as a factor in the acute phase reaction (Akira et al., 1994). After induction by IL-6, STAT3 acts as a transcriptional factor for many pro-inflammatory genes, including ICAM-1 (Heinrich et al., 2003). We also demonstrated that ${ }^{8}{ }^{8} d G$ suppresses the transcriptional level of ICAM-1 through STAT3 (Figure 3B, C). Our results have demonstrated that ICAM-1 expression is perfectly suppressed in DN-STAT3 expressing cells, indicating that STAT3 is an important molecule for ICAM-1 transcriptional expression. We also evaluated the in vivo effects of $\mathrm{oh}^{8} \mathrm{dG}$ on the interaction of STAT3 with the ICAM-1 promoter regions (Figure $3 D, E)$. The amount of this ChIP complex was dramatically reduced by $\mathrm{oh}^{8} \mathrm{dG}$. Thus, STAT3 proteins probably play crucial roles in regulating ICAM-1 gene promoter activity in LPS treated brain microglial cells.

Microglia, a major immune cell population in the central nervous system (CNS), are inactive in the healthy adult CNS, but become activated in acute and chronic neurodegenerative diseases (Kreutzberg et al., 1996). LPS is a common inflammogen used to activate microglia in several animal models of inflammation-mediated neurodegeneration, including both in vivo and in vitro models (Gao et al., 2002). The Toll-like receptor family is a diverse group of receptors that are responsible for recognition of microglial pathogens and protein patterns. This reaction is critical to the innate immune response (Means et al., 2000). TLR4 is traditionally accepted as the primary (LPS) receptor (Lien et al., 2000) and has been reported to be critical for the microglial response to LPS (Lehnardt et al., 2002). It has been reported that LPS is effective in rapidly up-regulating the expression of the endothelial ICAM-1 and the vascular cell-adhesion molecule (VCAM) (Bell et al.,
1995). Our results show that STAT3 contributes to LPS- induced inflammatory responses and $\mathrm{oh}^{8} \mathrm{dG}$ inhibited LPS-enhanced STAT3 activation in brain microglia. We also observed by MMT assay that $\mathrm{oh}^{8} \mathrm{dG}$ was not toxic (Figure 1A), indicating that the anti- inflammatory action of $\mathrm{oh}^{8} \mathrm{dG}$ is due to a mechanism underlying inhibition of STAT3 inflammatory signaling. However, the side effects of $\mathrm{oh}^{8} \mathrm{dG}$ in the cytosol are a concern. Therefore, after confirming the pharmacologic effects, a toxicological study of oh $^{8} \mathrm{dG}$ should be performed.

Although the signals that activate microglia in vivo are defined incompletely, several studies using in vitro models have shown that microglia respond to cytokines (IL-1 $\beta$, TNF- $\alpha$ and IFN- $\gamma$ ), microbial products, such as bacterial LPS, and certain diseaserelated abnormal proteins, including $\beta$-amyloid, human immunodeficiency virus (HIV)-specific gp41 and HIV-Tat, prions (Nakamura et al., 2002), and heat shock proteins (Kakimura et al., 2002). These factors activate their receptors and activate intracellular signaling pathway. The initial in vivo reports using TLR4-/- mice demonstrated a reduced susceptibility to sepsis with systemic administration of LPS (Poltorak et al., 1998). Early in vitro work with cells derived from TLR4-/- mice demonstrated that TLR4-/monocytes have an attenuated LPS cytokine response (Hoshino et al., 1999), indicating the critical role of TLR4 in the LPS-induced production of pro-inflammatory factors. However, while TLR4 is known to initiate the effects of LPS through a common and primary pathway, several other receptors have been identified in the induced effects of LPS, and there is a clear TLR4- independent response to LPS in phagocytes (Perera et al., 2001). For confirmation, the role of TLR4 in LPS-induced microglial activation was tested with mutant mice. Immunohistochemical staining of microglia with antibodies against OX-42 and STAT3 was used in brain tissue treated with LPS. Absence of functional TLR4 mice microglia did not staining with antibodies against OX-42 and STAT3. In our study, TLR4 was found to be indispensable for triggering a STAT3 pathway from LPS leading to activation of microglia.

Our results demonstrate that ${ }^{8}{ }^{8} \mathrm{dG}$ plays a role in anti-inflammatory actions via suppression of ICAM-1 gene expression by blockade of the TLR4-STAT3 signal cascade in brain microglia. Therefore, $\mathrm{oh}^{8} \mathrm{dG}$ in the cytosol probably functions as an anti-inflammatory molecule that will be useful as a therapeutic agent for neurodegenerative diseases.

\section{Acknowledgement}

This work was supported by grant no 800-20030142 from the Seoul National University Hospital Research 
Fund, and the Ministry of Science and Technology of Korea through the National Research Laboratory for Free Radicals.

\section{References}

Ahluwalia J, Tinler A, Clapp LH, Duchen MR, Abramov AY, Pope S, Nobles M, Segal AW. The large-conductance $\mathrm{Ca}^{2+}$ activated $\mathrm{K}^{+}$channel is essential for innate immunity. Nature 2004;427:853-8

Akira S, Nishio Y, Inoue M, Wang XJ, Wei S, Matsusaka T, Yoshida K, Sudo T, Naruto M, Kishimoto T. Molecular cloning of APRF, a novel IFN-stimulated gene factor 3 p91-related transcription factor involved in the gp130-mediated signaling pathway. Cell 1994;77:63-71

Baik SC, Youn HS, Chung MH, Lee WK, Cho MJ, Ko GH, Park $\mathrm{CK}$, Kasai $\mathrm{H}$, Rhee $\mathrm{KH}$. Increased oxidative DNA damage in Helicobacter pylori-infected human gastric mucosa. Cancer Res 1996;56:1279-82

Bell MD, Perry VH. Adhesion molecule expression on murine cerebral endothelium following the injection of a proinflammagen or during acute neuronal degeneration. J Neurocytol 1995;24:695-710

Butcher EC, Picker LJ. Lymphocyte homeing and homeostasis. Science 1996;272:60-6

Caldenhoven E, Coffer P, Yusan J, Van de Stolpe A, Horn F, Kruijer W, Van der Saag P. Stimulation of the human intercellular adhesion molecule-1 promoter by interleukin- 6 and interferon-gamma involves binding of distinct factors to a palindromic response element. J. Biol. Chem 1994;269:21146-54

Choi J, Yoon BJ, Han YN, Lee KT, Ha J, Jung HJ, Park HJ. Antirheumatoid arthritis effect of Rhus verniciflua and of the active component, sulfuretin. Planta Med 2003;69:899-904

Chung MH, Kasai H, Nishimura S, Yu BP. Protection of DNA damage by dietary restriction. Free Radic Biol Med 1992; $12: 523-5$

Dryden GW, Deaciuc I, Arteel G, McClain CJ. Clinical implications of oxidative stress and antioxidant therapy. Curr Gastroenterol Rep 2005;7:308-16

Fraga CG, Motchnik PA, Wyrobek AJ, Rempel DM, Ames BN. Smoking and low antioxidant levels increase oxidative damage to sperm DNA. Mutat Res 1996;35:199-203

Gao HM, Jiang J, Wilson B, Zhang W, Hong JS, Liu B. Microglial activation-mediated delayed and progressive degeneration of rat nigral dopaminergic neurons: relevance to Parkinson's disease. J Neurochem 2002;81:1285-97

Heinrich PC, Behrmann I, Haan S, Hermanns HM, MullerNewen G, Schaper F. Principles of interleukin (IL)-6-type cytokine signaling and its regulation. Biochem J 2003;374:1-20

Heneka MT, Dumitrescu L, Loschmann PA, Wullner U, Klockgether T. Temporal, regional, and cell-specific changes of iNOS expression after intrastriatal microinjection of interferon gamma and bacterial lipopolysaccharide. J Chem Neuroanat 2000;18:167-79

Hoshino K, Takeuchi O, Kawai T, Sanjo H, Ogawa T, Takeda
Y, Takeda K, Akira S. Cutting edge: Toll-like receptor 4 (TLR4)-deficient mice are hyporesponsive to lipopolysaccharide: evidence for TLR4 as the Lps gene product. J Immunol 1999;162:3749-52

Ihle JN. The stat family in cytokine signaling. Curr Opin Cell Biol 2001;13:211-7

Kakimura J, Kitamura Y, Takata K, Umeki M, Suzuki S, Shibagaki K, Taniguchi T, Nomura Y, Gebicke-Haerter PJ, Smith MA, Perry G, Shimohama S. Microglial activation and amyloid-beta clearance induced by exogenous heat-shock proteins. FASEB J 2002;16:601-3

Kasai $\mathrm{H}$. Analysis of a form of oxidative DNA damage, 8-hydroxy-2'-deoxyguanosine, as a marker of cellular oxidative stress during carcinogenesis. Mutat Res 1997;387:147-63

Kim HY, Park EJ, Joe EH, Jou I. Curcumin suppresses Janus kinase-STAT inflammatory signaling through activation of Src homology 2 domain-containing tyrosine phosphatase 2 in brain microglia. J Immunol 2003;171:6072-9

Kim JB, Yu YM, Kim SW, Lee JK. Anti-inflammatory mechanism is involved in ethyl pyruvate-mediated efficacious neuroprotection in the postischemic brain. Brain Res 2005;11: 188-92

Kim JE, Choi S, Too JA, Chung MH. 8-Oxoguanine induces intramolecular DNA damage but free 8-oxoguanine protects intermolecular DNA from oxidative stress. FEBS Lett 2004; 556:104-10

Kim JE, Chung MH. 8-oxo-7,8-dihydro-2'-deoxyguanosine is not salvaged for DNA synthesis in human leukemic U937 cells, Free Radic Res 2006;40:461-6

Kim OS, Park EJ, Joe EH, Jou I. JAK-STAT signaling mediates gangliosides-induced inflammatory responses in brain microglial cells. J Biol Chem 2002;277:40594-601

Kocak G, Aktan F, Canbolat O, Ozogul C, Elbeg S, YildizogluAri N, Karasu C. Alpha-lipoic acid treatment ameliorates metabolic parameters, blood pressure, vascular reactivity and morphology of vessels already damaged by streptozotocin diabetes. Diabetes Nutr Metab 2000;13:308-18

Kreutzberg GW. Microglia: a sensor for pathological events in the CNS. Trends neurosci 1996;19:312-8

Lehnardt S, Lachance C, Patrizi S, Lefebvre S, Follett PL, Jensen FE, Rosenberg PA, Volpe JJ, Vartanian T. The toll-like receptor (TLR4) is necessary for lipopolysaccharide-induced oligodendrocyte injury in the CNS. J Neurosci 2002;22: 2478-86

Lien E, Means TK, Heine H, Yoshimura A, Kusumoto S, Fukase K, Fenton MJ, Oikawa M, Qureshi N, Monks B, Finberg RW, Ingalls RR, Golenbock DT. Toll-like receptor 4 imparts ligand-specific recognition of bacterial lipopolysaccharide. J Clin Invest 2000;105:497-504

Liu B, Hong JS. Role of microglia in inflammation-mediated neurodegenerative diseases: mechanisms and strategies for therapeutic intervention. J Pharmacol Exp Ther 2003;304:1-7

Means TK, Golenbock DT, Fenton MJ. The biology of Toll-like receptors. Cytokine Growth Factor Rev 2000;11:219-32

Nakae D, Kobayashi Y, Akai H, Andoh N, Satoh H, Ohashi K, 
Tsutsumi M, Konishi Y. Involvement of 8-hydroxyguanine formation in the initiation of rat liver carcinogenesis by low dose levels of N-nitrosodiethylamine. Cancer Res 1997;57:1281-7

Nakamura Y. Regulating factors for microglial activation. Biol Pharm Bull 2002;25:945-53

Park C, Lee S, Cho IH, Lee HK, Kim D, Choi SY, Oh SB, Park $\mathrm{K}$, Kim JS, Lee SJ. TLR3-mediated signal induces proinflammatory cytokine and chemokine gene expression in astrocytes: Differential signaling mechanisms of TLR3-induced IP-10 and IL-8 gene expression. Glia 2006;53:248-56

Perera PY, Mayadas TN, Takeuchi O, Akira S, Zaks-Zilberman M, Goyert SM, Vogel SN. CD11b/CD18 acts in concert with CD14 and Toll-like receptor (TLR) 4 to elicit full lipopolysaccharide and taxol-inducible gene expression. J Immunol 2001;166:574-81

Poltorak A, He X, Smirnova I, Liu MY, Van Huffel C, Du X, Birdwell D, Alejos E, Silva M, Galanos C, Freudenberg M, Ricciardi-Castagnoli P, Layton B, Beutler B. Defective LPS signaling in $\mathrm{C} 3 \mathrm{H} / \mathrm{HeJ}$ and $\mathrm{C} 57 \mathrm{BL} / 10 \mathrm{ScCr}$ mice: mutations in TIr4 gene. Science 1998;282:2085-8

Shibutani S, Takeshita M, Grollman AP. Insertion of specific bases during DNA synthesis past the oxidation-damaged base 8-oxodG. Nature 1991;349:431-4

Shimosawa T, Fujita T. Adrenomedullin as a potent antioxidative and antiatherosclerotic substance. Drug News Perspect 2005;18:185-9
Simon AR, Rai U, Fanburg BL, Cochran BH. Activation of the JAK-STAT pathway by reactive oxygen species. Am J Physiol 1998;275:1640-52

Stratowa C, Audette M. Tanscriptional regulation of the human intercellular adhesion molecule-1 gene: a short overview. Immunobiology 1995;193:293-304

Wahl SM, Feldman GM, McCarthy JB. Regulation of leukocyte adhesion and signaling in inflammation and disease. J Leuk Biol 1996;59:789-96

Wilson VL, Taffe BG, Shields PG, povey AC, Harris CC. Detection and quantification of 8-hydroxydeoxyguanosine adducts in peripheral blood of people exposed to ionizing radiation. Environ Health Perspect 1993;99:261-3

Wu AJ, Chen ZJ, Kan EC, Baum BJ. Interferon-gamma-induced JAK2 and STAT1 signalling in a human salivary gland cell line. J Cell Physiol 1997;173:110-4

Yang XP, Irani K, Mattagajasingh S, Dipaula A, Khanday F, Ozaki M, Fox-Talbot K, Baldwin WM 3rd, Becker LC. Signal transducer and activator of transcription 3alpha and specificity protein 1 interact to upregulate intercellular adhesion molecule-1 in ischemic-reperfused myocardium and vascular endothelium. Arterioscler Thromb Vasc Biol 2005;25:1395-400

Ye SK, Agata Y, Lee HC, Kurooka H, Kitamura T, Shimizu A, Honjo T, Ikuta K. The IL-7 receptor controls the accessibility of the TCR locus by Stat5 and histone acetylation. Immunity $2001 ; 15: 813-23$ 